\title{
The Influence of Face Masks on Verbal Communication in Persian in the Presence of Background Noise in Healthcare Staff
}

\author{
Mohsen Aliabadi $^{1}$ (D) Zahra Sadat Aghamiri $^{1} \cdot$ Maryam Farhadian $^{2} \cdot$ Masoud Shafiee Motlagh $^{1}$. \\ Morteza Hamidi Nahrani ${ }^{3}$
}

Received: 9 October 2021 / Accepted: 21 November 2021 / Published online: 26 January 2022

(c) Australian Acoustical Society 2021

\begin{abstract}
Wearing face masks has resulted in verbal communication being more challenging during the COVID-19 pandemic. This study aimed to investigate the effect of face masks on the speech comprehensibility of Persian nurses in healthcare settings. Twenty female nurses from the governmental hospitals randomly participated in an experiment on seven typical commercial face masks at two background noise levels. Nurses' speech intelligibility from a human talker when wearing each face mask was determined based on the speech discrimination score. The vocal effort of nurses wearing each face mask was determined based on the Borg CR10 scale. Based on the linear mixed model, the speech intelligibility of nurses from a human speaker wearing surgical masks, N95 masks, and a shield with face masks were approximately 10\%, 20\%, and 40-50\% lower, respectively, than no-mask conditions $(p<0.01)$. The background noise decreased the speech intelligibility of nurses by approximately $22 \%(p<0.01)$. The use of a face shield further decreased speech intelligibility up to $30 \%$ compared to using a face mask alone $(p<0.01)$. The vocal efforts of nurses when wearing surgical masks were not significant compared with the baseline vocal efforts $(p>0.05)$; however, vocal efforts of nurses when wearing N95 and N99 respirators were at an unacceptable level. The face masks had no considerable effect on the speech spectrum below $2.5 \mathrm{kHz}$; however, they reduced high frequencies by different values. Wearing face masks has a considerable impact on the verbal communication of nurses in Persian. The level of background noise in the healthcare setting can aggravate the effect sizes of face masks on speech comprehensibility.
\end{abstract}

Keywords Verbal communication $\cdot$ Face mask $\cdot$ Background noise $\cdot$ Healthcare setting $\cdot$ Speech intelligibility

\section{Introduction}

Face masks or face shields are commonly used in healthcare settings by healthcare staff to prevent contact and respiratory exposure to patient discharge, droplets, and airborne particles. Wearing face masks also decreases the transmission of respiratory droplets containing hazardous viruses, especially during the COVID-19 pandemic [1]. If for some reason the

Mohsen Aliabadi

mohsen.aliabadi@umsha.ac.ir; mohsen_ohse@yahoo.com

1 Center of Excellence for Occupational Health, School of Public Health and Occupational Health and Safety Research Center, Hamadan University of Medical Sciences, P.O. Box 4171-65175, Hamadan, Iran

2 Department of Biostatistics, School of Public Health and Research Center for Health Sciences, Hamadan University of Medical Sciences, Hamadan, Iran

3 Department of Audiology, School of Rehabilitation, Hamadan University of Medical Sciences, Hamadan, Iran medical staff has to work even for a short time without a mask, there is a possibility that the risk of exposure is increased. Among the reasons that lead to the intermittent use of the mask is the effect of the face mask on the speech ability of the speaker and impaired understanding of the speech by the audience $[1,2]$. Frequent wearing and removing of the face mask can also increase the likelihood of its surface contamination [3]. Face masks can have detrimental effects on speech intelligibility and verbal communication by covering main visual cues from lip and mouth gestures, interfering with oral joint movements, and changing speech acoustic characteristics. The social distancing rule along with wearing face masks has resulted in verbal communication being more challenging [2].

Communication is an essential part of nursing care and practice. Clear conversation and speech intelligibility are especially crucial for nurses during medical emergency conditions. There is some concern that face coverage may have a negative effect on the speech comprehensibility of nurses in 
hospital settings, which can also lead to irreparable medical errors [2]. Hospital environments usually have noise pollution caused by traffic, equipment passage, and the presence of patients, which in itself can affect the speech of medical staff. Nickell et al. [4] showed that background noise levels in Toronto hospitals increase to 72 and $60 \mathrm{dBA}$ during the daytime and the nighttime, respectively. Due to the high background noise levels, $47 \%$ of the hospitals' staff noted some difficulties in verbal communication related to wearing facial respirators. It should be noted that the World Health Organization recommends a noise exposure limit up to $40 \mathrm{dBA}$ for the day shift and $35 \mathrm{dBA}$ for the night shift in hospitals [4]. A systematic review by Andrade et al. [5] showed that in the hospital environments located in Asia, Europe, and the Americas, the background noise levels have been reported ranging from 37 to $88.6 \mathrm{dBA}$ for daytimes and ranging from 38.7 to $68.8 \mathrm{dBA}$ for nighttime. Darbyshire and Young [6] showed that the background noise levels of intensive care units (ICUs) are frequently greater than $45 \mathrm{dBA}$, with more than half of the daytime from 52 to $59 \mathrm{dBA}$. Pai [7] also showed that the background noise levels in the hospitals' stations and wards were from 50.3 to $68.1 \mathrm{dBA}$ which is above the recommended exposure noise limit. The signal-to-noise ratio in an indoor environment is another critical factor affecting speech intelligibility. However, the effect of face masks on the ability to transmit speech at different signal-to-noise ratios has been less studied.

Some different commercial face masks have been widely used during the pandemic by healthcare staff in Iranian healthcare settings, but their acoustic characteristics have not been as widely studied. It is essential to determine the potential effects of wearing these face masks on speech intelligibility in typical hospitals with significant background noise levels which can pose a major challenge in staffs' verbal communication.

Corey et al. [8] showed that surgical masks were found to have little impact on speech intelligibility. Surgical masks have especially mild acoustic effects. The results of another study by Corey et al. [9] reported that there are fundamental differences among face masks in terms of acoustic properties. Chmelik et al. [10] reported that when people wear masks, they tend to raise their voice level. Yi et al. [11] indicated that in the presence of background sound, the listeners had a lower performance than the voices of speakers wearing face masks compared to no-mask condition. Wearing face masks by the speaker in the presence of background noise can affect the comprehension of audio signals for listeners. They proposed that some studies should include different background noise levels to describe a diversity of common communicative situations. Wittum [12] showed that the correct percentage of sentence recognition was high for the no-mask condition, $33.1 \%$ for the conditions of wearing a mask, and $20.9 \%$ for wearing a face mask plus blood shield. Randazzo et al. [13] stated that all face masks reduce speech signals, especially N95 masks with the greatest overall effect.

Vocal effort is defined as the perceived exertion during voicing or speaking. Speech requires negligible vocal effort in humans with normal vocal mechanisms in environments with low background noise. Speech effort usually increases in response to certain environmental factors, including overcoming high ambient noise as well as sound propagation over long distances [14]. Wearing face masks can also raise the vocal effort of the speaker in the form of an effect on the voice/breathing coordination, changes the perceptual characteristics of the voice, and increases vocal track discomfort levels. The use of a mask increases the feeling of verbal discomfort, especially in staff who wear them for daily job activities [15]. The vocal effort is mainly a subjective or perceived response, hence, the Borg CR10 scale can be employed as a helpful psychometric scale for determining it. Leer and Mersbergen [16] verified the usability and validity of the Borg CR10 scale in determining the level of perceived vocal effort in the healthcare setting.

It can be stated that the consequences of available commercial face masks on verbal communication in the presence of background noise are extremely important in healthcare settings. As mentioned, less attention has been paid to the effect of face masks used by nurses on verbal communication in Persian compared to existing data about particle removal efficiency. The present study aimed to empirically investigate the effect of face masks on speech comprehensibility in simulated acoustic conditions of the Iranian healthcare settings. Determination of the effect sizes of the available face masks on the speech comprehension of healthcare staff can help professionals in selecting the appropriate face mask as well as applying new techniques in producing face masks with appropriate acoustic characteristics.

\section{Methods}

\subsection{Face Mask Samples}

In this study, as shown in Fig. 1, seven typical commercial face masks with technical specifications from reliable national manufacturers were investigated, including the two medical or surgical masks, two N95 respirators with a filter, three-dimensional (3D) mask, N99 respirators with a filter, and N99 respirators without a filter. Table 1 shows some important characteristics, including materials, layers, and surface density of the studied face masks. Most national industries use spun bond-melt blown-spun bond (SMS) technology for producing available surgical masks. The studied face masks are commonly composed of polypropylene spun bond and melt blown fabric laminate. For example, in surgical masks, the inner spun-bond nonwoven fabric layer absorbs 


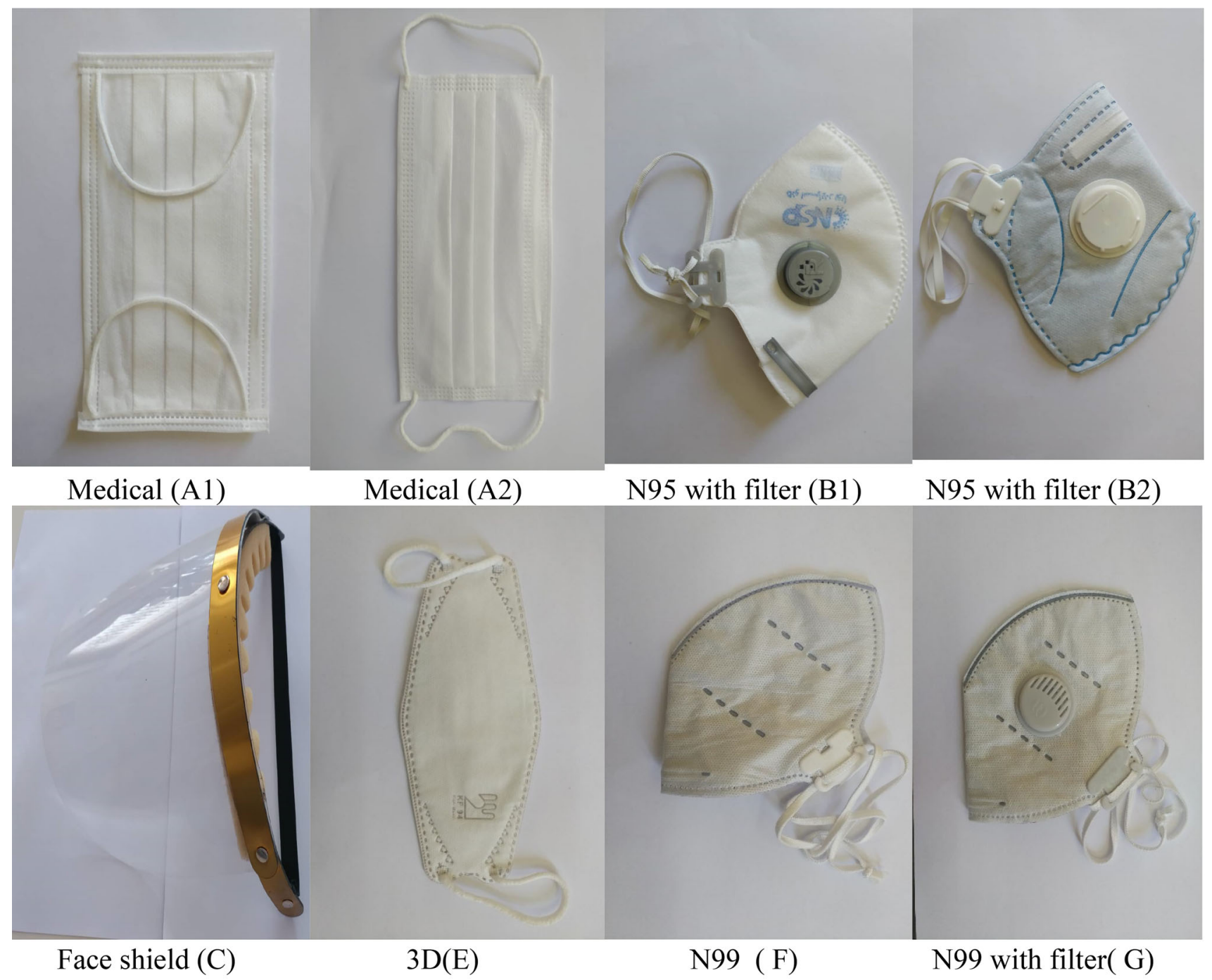

Fig.1 Appearance of the studied face masks along with identification codes

moisture released by the user, and the outer layer is mainly applied to create a barrier between the external droplets and the users. The middle melt-blown nonwoven fabric is the filter layer that provides protection from airborne pollutants. It should be noted that spun bond nonwoven fabrics are composed of continuous filaments produced by an integrated fiber spinning, web formation, and bonding process. The N95 and N99 respirators are comprised of many layers of polypropylene nonwoven fabric.

\subsection{Participants}

In this experimental study, 20 female nurses from hospitals affiliated with Hamadan University of Medical Sciences (located in the west of Iran) with an age range of 25-40 years were randomly selected. It should be noted that most of the medical staff in the Iranian healthcare settings are females; hence, all of the subjects who participated were considered female staff. Statistics from the Iranian Nursing Organization show that more than $80 \%$ of nurses in hospitals are women. Inclusion criteria for participating in the study included having normal hearing status (hearing threshold lower than $25 \mathrm{~dB}$ ). Pure tone audiometry was conducted to determine a person's hearing threshold using an audiometer (MAICO; model KS5). The audiometry test environment was an acoustic room with furnished wall surfaces. The test environment was according to the ISO 8253 [17] recommendations, an acoustic room to provide a background noise level lower than $30 \mathrm{dBA}$ and a reverberation time of lower than $0.5 \mathrm{~s}$ per frequency in the range of $125-8000 \mathrm{~Hz}$. This study was approved by the Ethics Committee of Hamadan University of Medical Sciences (ethic code: IR.UMSHA.REC.1399.320). After being informed about this study, each participant completed a consent form. The exclusion criteria for selecting 
Table 1 Design and material characteristics of the studied face masks

\begin{tabular}{|c|c|c|c|c|}
\hline Mask code & Type & Material & Surface density $\left(\mathrm{g} / \mathrm{m}^{2}\right)$ & Ply \\
\hline A1 & Medical mask & $\begin{array}{l}\text { (Spun bond })+(\text { melt blown })+ \\
\quad(\text { spun bond })\end{array}$ & 51 & 3 \\
\hline $\mathrm{A} 2$ & Medical mask & $\begin{array}{l}(\text { Spun bond }- \text { melt blown })+ \\
\quad(\text { spun bond }- \text { melt blown })\end{array}$ & 60 & 2 \\
\hline B1 & N95 respirator & $\begin{array}{l}\text { (Spun bond })+(\text { spun bond }- \\
\text { melt blown })+(\text { spun bond }- \\
\text { melt blown })+(\text { spun bond }- \\
\text { melt blown })+(\text { spun bond }- \\
\text { melt blown })\end{array}$ & 120 & 5 \\
\hline B2 & N95 respirator & $\begin{array}{l}\text { (Spun bond })+(\text { melt blown })+ \\
(\text { spun bond })+(\text { melt blown })+ \\
(\text { spun bond })+(\text { spun bond })\end{array}$ & 180 & 6 \\
\hline $\mathrm{E}$ & Three-dimensional & $\begin{array}{l}\text { (Spun bond }- \text { melt blown })+ \\
(\text { melt blown })+(\text { spun bond }- \\
\text { melt blown })+(\text { spun bond }- \\
\text { melt blown })\end{array}$ & 130 & 4 \\
\hline $\mathrm{F}$ & N99 respirator & $\begin{array}{l}(\text { Spun bond }- \text { melt blown })+ \\
(\text { spun bond }- \text { melt blown })+ \\
(\text { spun bond }- \text { melt blown })+ \\
(\text { spun bond })+(\text { spun bond })\end{array}$ & 145 & 5 \\
\hline G & N99 respirator & $\begin{array}{l}(\text { Spun bond }- \text { melt blown })+ \\
(\text { spun bond }- \text { melt blown })+ \\
(\text { spun bond }- \text { melt blown })+ \\
(\text { spun bond })+(\text { spun bond })\end{array}$ & 145 & 5 \\
\hline
\end{tabular}

participants included the presence of voice and tone disorders, respiratory disorder or infection, and any oral disability.

\subsection{Determination of Speech Intelligibility}

To measure the acoustic performance of the face mask in more realistic conditions, the speech from a human talker when wearing each face mask was recorded. According to the ISO 8253-3 [18] standard, a different series of accurate and reliable lists of one-syllable Persian words were used for reading by a human talker for each face mask. It should be noted that each series included 25 words. A female talker was selected as a human speaker who tried to preserve a consistent speech level during each voice recording. The talker avoided emphasizing words and maintained normal speed and clarity of speech during the recording of each scenario. To further ensure the production of the speaker's voice at a constant level in different masks, BANDICAM 4.6.5 recorder software was used to monitor the speech, and if there were fluctuations, the recording was repeated.

The speech was recorded at the mentioned acoustic studio to provide the least background noise level in the range of $125-8000 \mathrm{~Hz}$. A headset with a fixed microphone was used on the head of a human speaker when wearing face masks to record speech in the same conditions in all sessions. The speech intelligibility test was conducted based on the speech discrimination score (SDS). Nurses as listeners were positioned $2 \mathrm{~m}$ away from the loudspeakers without any movement or making noise or talking in an experiment room that simulated the acoustic characteristics of the typical hospital environment. So it can be expressed in the phase of the human talker recordings, a headset microphone was used to record speech as direct sound passing at the closest point to the surface of the face mask. In the next phase, the recorded speech affected by passing the face mask was played back for the listener positioned $2 \mathrm{~m}$ away from the speaker based on the social distancing rule during the pandemic.

The listeners were asked to listen for one-syllable Persian words recorded for each mask and write it. Then, the percentage of correct words repeated by the listeners was determined. Based on EC 60268-16 [19] standard recommendation, the percentage of syllable intelligibility was classified: bad 0-34\%; poor 34-48\%; fair 48-67\%; good 67-90\%; and excellent 90-96\%. The baseline speech intelligibility of each participant was determined at the acoustic studio at the lowest background noise level [20].

The speech intelligibility of participants for each face mask was determined at two ambient background noise levels of 60 and $70 \mathrm{dBA}$. The recorded speech stimuli were emitted at an approximately normal voice range of about $70 \mathrm{dBA}$ for providing an approximate signal-to-noise ratio of $0 \mathrm{~dB}$ at a background noise level of $70 \mathrm{dBA}$ and an approximate signal-to-noise ratio of $+10 \mathrm{~dB}$ at a background noise level of $60 \mathrm{dBA}$. Given that each face mask may have had a different quantitative effect on the speaker's voice, the signal value was not inherently fixed, but the background noise was con- 


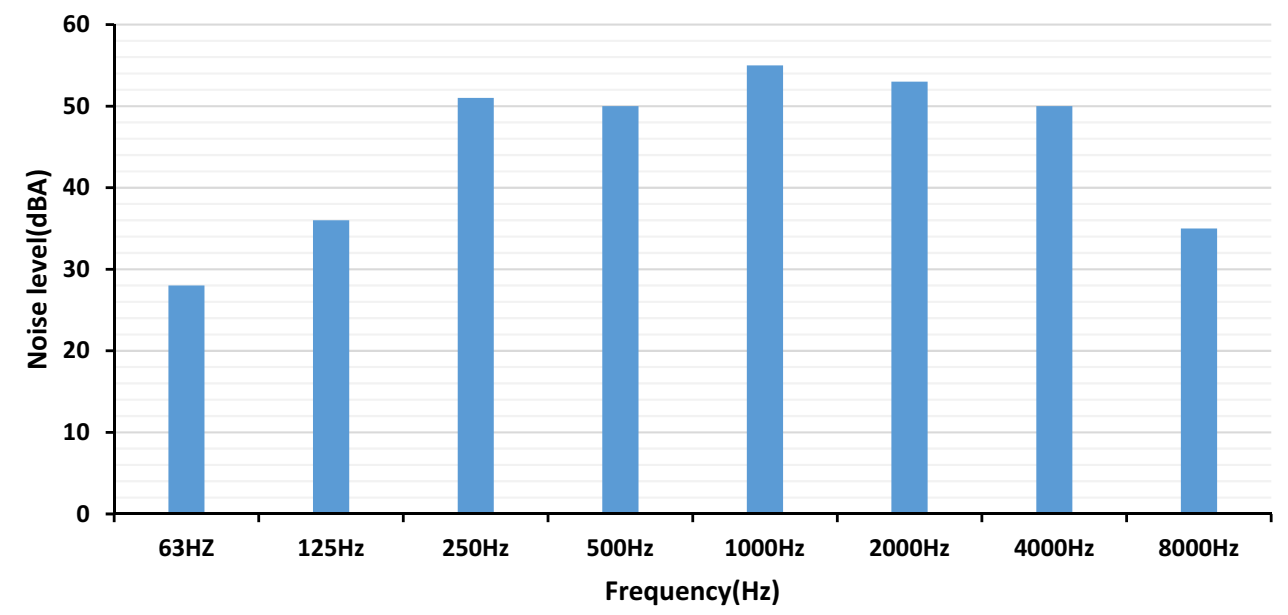

Fig. 2 Frequency spectrum of the typical background noise of a healthcare setting

stant. The background noise was recorded from a typical real workstation of healthcare staff at a hospital and simulated in a test environment using two loudspeakers (Pejvak Ava CO). The frequency spectrum of the recorded background noise in a healthcare setting is presented in Fig. 2. As shown, the dominant frequencies of the hospital background noise are in a medium-frequency range.

\subsection{Determination of Vocal Effort}

The Borg scale for rating perceived exertion, defined by Gunnar Borg, is a tool for determining exertion and effort level, fatigue, and breathlessness level during physical activities $[14,16,21]$. This scale is successfully used in various fields of medicine, ergonomics, and kinesiology to determine the level of perceived exertion and effort. The scale is a very simple numerical list, as shown in Table 2. In this study, each participant read the prepared standard text continuously for 3 minutes while wearing each face mask with background noise recorded from typical real healthcare workstations. The background noise levels of 70 and $75 \mathrm{dBA}$ were played using loudspeakers in the experiment room. Based on the literature, these background noises are common examples of noisy hospital settings that increase the need for speech effort, and as a result, the effect of different types of face masks on vocal effort can be better determined and compared. The baseline vocal effort of each participant was determined at the acoustic studio with the least background noise level. Participants were requested to rate their effort and exertion on the scale after the defined reading activity when wearing each face mask so that it can combine all feelings and sensations about perceived physical fatigue and stress.
Table 2 Borg CR10 scale for determination of vocal effort

\begin{tabular}{ll}
\hline Score & Level of exertion \\
\hline 0 & No exertion at all \\
0.5 & Very, very slight (just noticeable) \\
1 & Very slight \\
2 & Slight \\
3 & Moderate \\
4 & Somewhat severe \\
5 & Severe \\
6 & Very severe \\
7 & \\
8 & Very, very severe \\
9 & \\
10 & Maximal \\
\hline
\end{tabular}

\subsection{Measurement of Speech Spectrum Attenuation}

A head-shaped loudspeaker was used to measure the acoustic attenuation of each face mask for a distant listener. The face masks were mounted and fitted exactly on the face of the mannequin head as shown in Fig. 3. A small loudspeaker (Tesco, model TS 2385, sound power of $5 \mathrm{~W}$, frequency range of $100-18,000 \mathrm{~Hz}$ ) with dimensions of $60 \times 50 \times 60 \mathrm{~mm}$ mounted and fully sealed within the mouth of the mannequin head. An aperture with dimensions of $6 \times 30 \mathrm{~mm}$ at the lip allowed the head to emit speech and noise signals. The head mannequin was made of epoxy resin material so that there was no vibration and sound transmission from its body except at the lip opening. The inside of the mannequin was filled with absorbent material made of polyurethane foam. The mannequin head was placed on a microphone stand at 

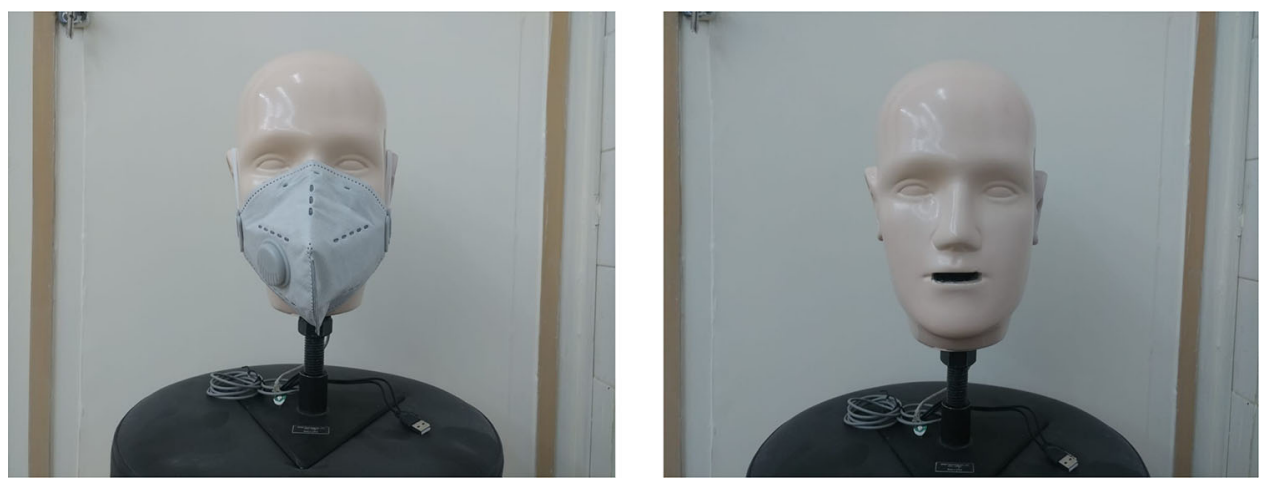

Fig. 3 A head-shaped loudspeaker with and without face mask

a standard height of $1.8 \mathrm{~m}$ in the middle of the room away from the wall and floor surfaces to reduce possible sound reflections. In the experiments using the artificial head, a 30$\mathrm{s}$ audio recording of a normal voice in Persian was played through the loudspeaker in the mouth lips, simulating artificial speech. To measure the frequency response of each mask for speech signal, a reference sound level meter was placed $1.8 \mathrm{~m}$ away from the mannequin head, such that it pointed axially at the lips. The distance of $1.8 \mathrm{~m}$ was chosen because it represents a safe distance for following the pandemic health protocols. The measurement of the received speech signals was performed based on a time-weighted or equivalent noise spectrum in a one-third octave band using the sound level meter (Svanteck, SVAN 971; class 1).

\subsection{Statistical Analysis}

Data were analyzed in SPSS software (ver. 22, Chicago, IL, USA). Data normality was tested using the Kolmogorov-Smirnov test. The significant relation among some features was analyzed using Pearson correlation. Wilcoxon's tests were used when the data distribution was not normal. The statistical significance level for all tests was set at 5\%. A linear mixed model (LMM) was also run to analyze the acoustic effect of background noise and face masks on the Persian speech quality indicators.

\section{Results}

Descriptive statistics of the speech intelligibility of nurses from a human speaker wearing different face masks in the presence of background noise are shown in Fig. 4. The results showed that the baseline speech intelligibility of the nurses was $98.80 \pm 1.24 \%$. As shown in Fig. 4, the findings descriptively revealed the speech intelligibility of nurses from a human speaker wearing a face mask decreases compared to the no-mask condition in the presence of background noise.
The speech intelligibility of nurses from a human speaker wearing the surgical and N95 face masks were at a fair level according to EC 60268-16 (2020) standard classifications. However, the speech intelligibility from a human speaker wearing a face mask along with a shield was from bad to poor.

The effects of different background noise and face masks on the speech intelligibility of nurses based on the estimated marginal means acquired by the LMM model are shown in Table 3. It should be noted that the speech intelligibility for each mask was considered based on the estimated marginal means at both background noise levels. The results showed that the background noise decreased the speech intelligibility of nurses by approximately $22 \%$ compared with the baseline value $(p<0.01)$. The results also showed the speech intelligibility of nurses from a human speaker wearing different face masks at the background noise level of $70 \mathrm{dBA}$ is approximately $15 \%$ lower than the background noise level of $60 \mathrm{dBA}$ $(p<0.01)$.

The speech intelligibility of nurses from a human speaker wearing surgical masks, N95 masks with filter, and face masks along with shield are approximately $10 \%, 20 \%$, and $40-50 \%$, respectively, less than when not using face masks in the presence of background noise levels $(p<0.01)$. The results showed speech intelligibility of nurses from a human speaker wearing an N95 face mask with a filter is approximately $10 \%$ lower than when using surgical face masks in the presence of background noise levels $(p<0.01)$. The results showed the use of a face shield further decreases the speech intelligibility of nurses from 25 to $30 \%$ compared to using face masks alone in the presence of background noise levels $(p<0.01)$. No interaction was observed between background noise levels and face masks with respect to speech intelligibility $(p>0.05)$.

Descriptive statistics of the vocal effort of nurses for face masks in the presence of background noise levels are presented in Fig. 5. The effects of different background noise levels and face masks on vocal effort scores based on the 


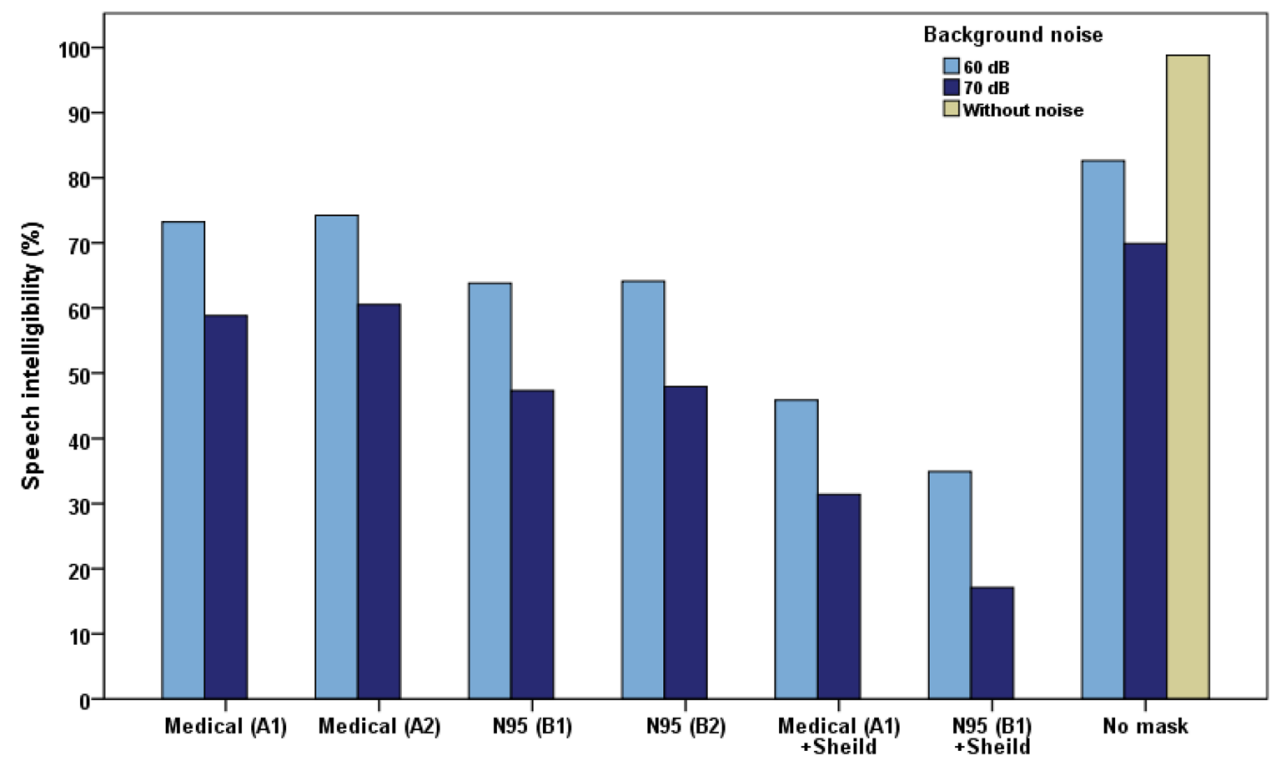

Fig. 4 Descriptive statistics of the speech intelligibility of nurses for face masks in the presence of background noise levels

Table 3 The effects of different background noise levels and face masks on speech intelligibility based on the linear mixed model

\begin{tabular}{lllll}
\hline Parameter & Estimate & SE & $p$ value & Estimated marginal means \pm SE \\
\hline Intercept & 98.80 & 1.24 & 0.000 & - \\
A1 & -11.10 & 1.48 & 0.000 & $66.00 \pm 1.00$ \\
A2 & -9.40 & 1.48 & 0.000 & $67.35 \pm 1.00$ \\
B1 & -22.60 & 1.48 & 0.000 & $55.55 \pm 1.00$ \\
B2 & -22.00 & 1.48 & 0.000 & $56.00 \pm 1.00$ \\
A1 + shield & -38.50 & 1.48 & 0.000 & $38.65 \pm 1.00$ \\
B1 + shield & -52.80 & 1.48 & 0.000 & $26.00 \pm 1.00$ \\
No mask (reference) & 0 & 0 & - & $76.25 \pm 0.90$ \\
60 dBA & -16.200 & 1.48 & 0.000 & $62.67 \pm 0.77$ \\
70 dBA & -28.90 & 1.48 & 0.000 & $47.55 \pm 0.77$ \\
Baseline & 0 & 0 & - & $98.80 \pm 1.24$ \\
\hline
\end{tabular}

estimated marginal means acquired by the LMM model are shown in Table 4. It should be noted that the vocal effort for each mask was considered based on the estimated marginal means at both background noise levels.

Overall, the baseline vocal efforts of nurses were much lower than very slight exertion without using a face mask and no background noise. However, in the presence of higher background noise, the vocal efforts of nurses without using a mask were increased to within very slight exertion. The vocal efforts of nurses when wearing surgical face masks were increased from very slight exertion to slight exertion which was not statistically significant compared with the baseline vocal efforts $(p>0.05)$. The vocal efforts of nurses when wearing three-dimensional face masks were increased from slight to moderate exertion and were statistically significant compared with the baseline vocal efforts $(p<0.05)$. The vocal efforts of nurses when wearing N95 face masks with the filter were increased up to severe exertion. However, the N99 face mask with a filter imposed lower verbal exertion compared to the N99 face mask without the filter. Generally, the vocal efforts of nurses when wearing N95 and N99 respirators are at an unacceptable level. In this case, nurses feel physical stress and fatigue during continuous conversation or lecture. The results also showed that in the use of all face masks, increasing the background noise by $5 \mathrm{~dB}$ increases the vocal effort of nurses from moderate to somewhat severe exertion. No interaction was observed between background noise levels and face masks with respect to vocal effort ( $p$ $>0.05)$.

Figure 6 shows that the effect of face masks on the speech spectrum at a one-third octave band at the listener position with respect to no-mask condition. The results showed the average high-frequency attenuation of speech signals for all face masks. In this experiment, the face masks had little effect 


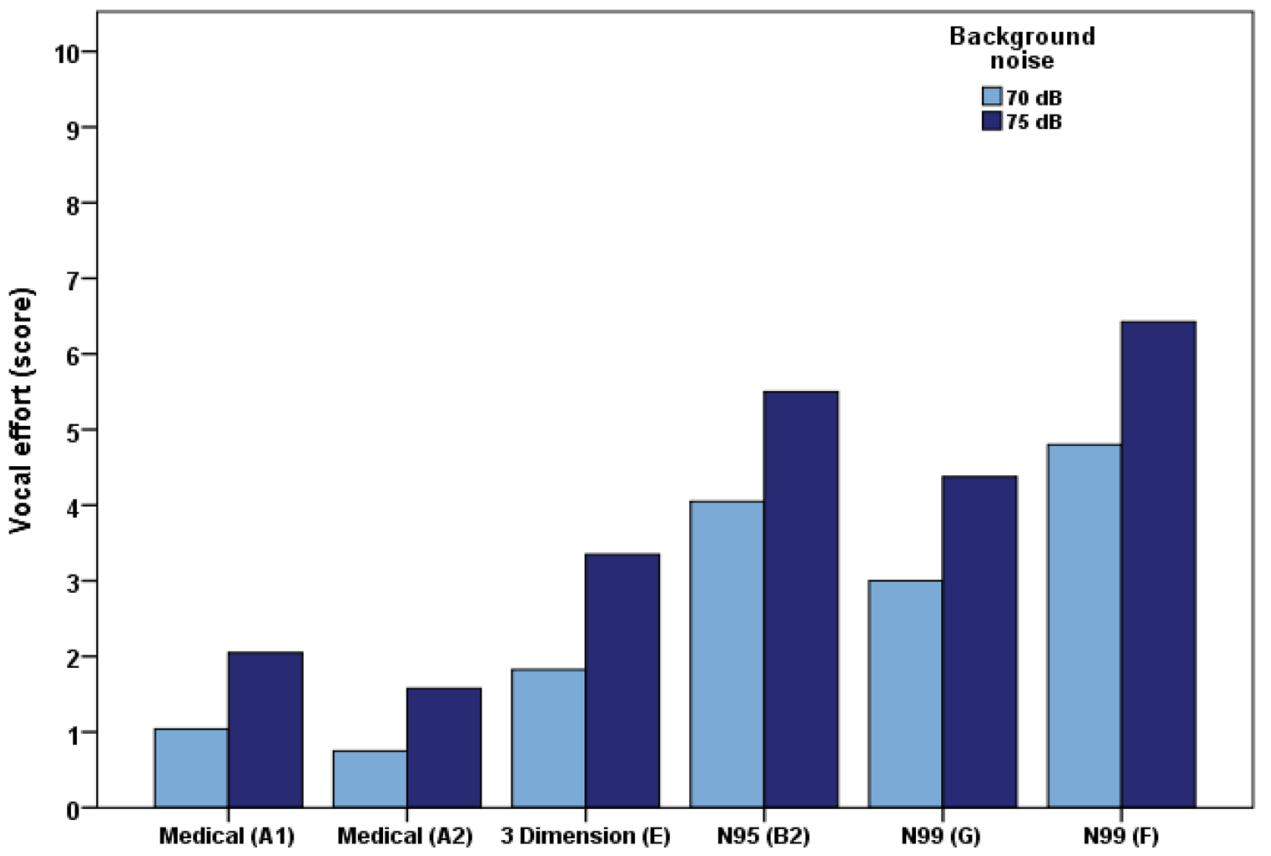

Fig. 5 Descriptive statistics of the vocal effort for face masks in the presence of background noise levels

Table 4 The effects of different face masks and background noise levels on vocal effort based on the linear mixed model

\begin{tabular}{lllll}
\hline Parameter & Estimate & SE & $p$ value & Estimated marginal mean \pm SE \\
\hline Intercept & 6.42 & 0.25 & 0.000 & - \\
A1 & -4.37 & 0.25 & 0.000 & $1.54 \pm 0.22$ \\
A2 & -4.85 & 0.25 & 0.000 & $1.16 \pm 0.22$ \\
E & -3.07 & 0.25 & 0.000 & $2.58 \pm 0.22$ \\
B2 & -0.92 & 0.25 & 0.000 & $4.77 \pm 0.22$ \\
G & -2.05 & 0.25 & 0.000 & $3.68 \pm 0.22$ \\
F & -2.05 & 0.25 & 0.000 & $5.61 \pm 0.22$ \\
No mask (reference) & 0 & 0 & - & $1.05 \pm 0.22$ \\
70 dBA & -16.200 & 1.48 & 0.000 & $2.57 \pm 0.195$ \\
75 dBA & -11.32 & 1.48 & 0.000 & $3.87 \pm 0.195$ \\
Baseline (reference) & 0 & 0 & - & $0.72 \pm 0.195$ \\
\hline
\end{tabular}

on speech signals below $2000 \mathrm{~Hz}$, and they showed high sound attenuation above $2500 \mathrm{~Hz}$ at a one-third octave band. Generally, most studied face masks had no considerable effect below $2.5 \mathrm{kHz}$, but they attenuated higher frequencies by different amounts. The greatest sound attenuation was approximately observed at the central frequency of 5000 to $6300 \mathrm{~Hz}$ for N95 and N99 respirators. The greatest sound attenuation was observed at the central frequency of $8000 \mathrm{~Hz}$ for surgical masks. Generally, the effect of surgical masks on reducing the transmission of speech spectrum is insignificant compared to N95 and N99 respirators.

\section{Discussion}

The COVID-19 pandemic has extremely influenced interpersonal communication, especially in healthcare settings. Face masks are essential for healthcare staff, but characteristics of the speech signal in Persian whilst wearing these respiratory devices are not experimentally reported. This study tried to explore new evidence about the role of face masks and background noise on Persian speech comprehensibility during interpersonal communication. 


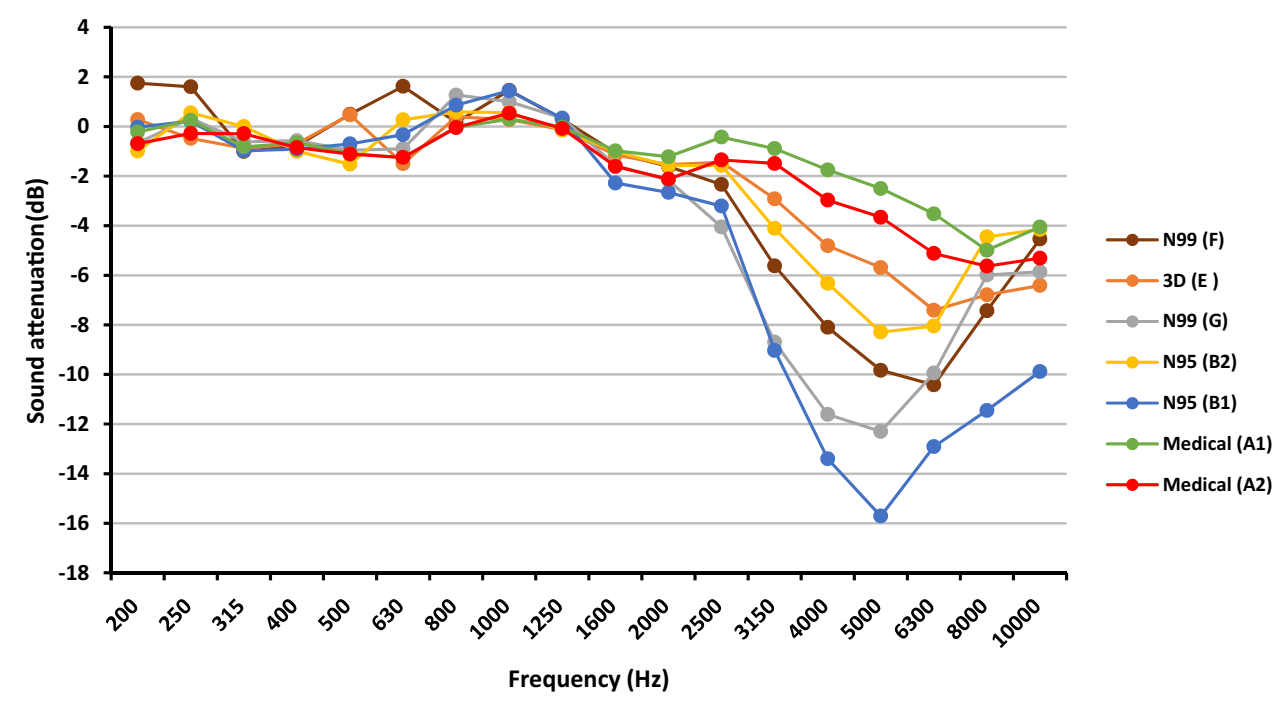

Fig. 6 Effect of face masks on the speech spectrum at the listener position with respect to no-mask condition

The current results demonstrated that different face masks affect the speech intelligibility of nurses depending on the level of background noise. Based on the developed LMM, the speech intelligibility of nurses from a human speaker wearing face masks is significantly lower than no-mask condition. However, the speech intelligibility of nurses from a human speaker wearing the surgical and N95 face masks is at a fair level. Therefore, it can be stated that surgical face masks have no detrimental effect on the speech communication of nurses. Our finding is similar to the results reported by Fiorella et al. [22], who showed that using a surgical mask does not create a significant effect on acoustic voice parameters. Toscano [23] showed that in low background noise levels, surgical face masks had no considerable effect, reducing the accuracy of word recognition by a maximum of $5.5 \%$ compared to a nomask condition. In high background noise, the percentage of correct word recognition decreased between 2.8 and 18.2\% compared to no-face mask conditions. However, the surgical masks showed no significant difference in any of the tested conditions. Their study suggests that the surgical mask is preferred for verbal communication if high levels of background noise are predicted. However, surgical masks are relatively sufficient for protecting public users in the high space area with possible proper social distancing. In healthcare settings, surgical masks are commonly employed to block large respiratory droplets during routine surgical procedures but do not filter small particles [24]. Moreover, wearing a surgical mask can help limit the spread of some respiratory particles by patients, so they are effective at preventing virus spread. Hence, N95 and N99 respirators are usually advised to protect the health of nurses or doctors around patients who are potentially infected with respiratory viruses [25].
This means that if the background noise is a bit loud, the N95 mask will keep the speech transmission in optimal condition to some extent. In this regard, JiChoi [26] also reported the intelligibility scores obtained from young adult listeners wearing N95 respirators reduced to a maximum of $10 \%$ at the signal-to-noise ratio of $5 \mathrm{~dB}$ compared to the results acquired in no-mask conditions. Moreover, the perceived effort ratings by participants wearing N95 respirators increased to a maximum of $10 \%$ at low background noise compared to the results acquired in no-mask conditions.

It is observed that the speech intelligibility of nurses from a human speaker wearing a shield along with a face mask is from bad to poor. Atcherson et al. [27] also showed that although transparent masks do not have the problem of speech communication through lip and mouth movements, they further attenuate speech transmission compared to regular face masks. Homans and Vroegop [28] showed that the face shield had a negative effect on speech intelligibility, in that its speech distortion was greater than that with the surgical mask alone.

The findings verified that an increase in background noise decreased the speech intelligibility of nurses considerably compared with the baseline value. Toscano [23] also demonstrated that differences in acoustic performance among face masks become more obvious in high background noise. Smiljanic et al. [29] showed that under normal environmental conditions, in terms of background sound, face masks have less effect on recognizing spoken words compared to high background noise conditions. The current results showed the average high-frequency attenuation of speech signals for all face masks at a one-third octave band greater than $2.5 \mathrm{kHz}$. Our finding is similar to the results reported by Corey et al. [9] who showed face masks had little effect on sound below 
$1 \mathrm{kHz}$, modest attenuation between 1 and $4 \mathrm{kHz}$, and strong attenuation above $4 \mathrm{kHz}$. Chmelik et al. [10] also found that all face shields and masks attenuate frequencies above 1-2 kHz. Magee et al. [1] reported significant differences in acoustic power distribution across relevant frequency bands for speech in all tested masks compared to no mask. However, the differences were not observed in frequencies below $3 \mathrm{kHz}$. The current findings verified that the effect of surgical masks on decreasing the transmission of the voice spectrum is insignificant compared to N95 and N99 respirators. JiChoi [26] showed that the surgical and KF94 face masks perform better acoustically at high frequencies between 2 and $5 \mathrm{kHz}$ than the N95 respirator.

The vocal efforts of nurses when wearing surgical face masks were not significant compared with the baseline vocal efforts; however, vocal efforts of nurses when wearing N95 and N99 face masks are at an unacceptable level. The results also showed that increasing the background noise increases the vocal effort of nurses when wearing different face masks. In this regard, Karagkouni [30] showed that the face masks increase the vocal effort of the speaker and disturb the voice/breathing coordination results in vocal discomfort levels.

The interpretation of the current results is limited to the tested commercial face masks and defined background noise in some nursing service workstations. Further studies should be undertaken to seek a balance between face mask protection and the ability to verbally communicate.

In the current study, the speech from a human talker when wearing each face mask was recorded, and then the speech was played back over loudspeakers for listeners. As noted, wearing a face mask has a noticeable effect on the main visual cues from lip and mouth gestures of the speaker, so there is no significant difference in face-to-face and offline conversation when wearing a face mask. The recorded background noise from real healthcare was emitted in a test environment using two loudspeakers. The simulated background noise condition using a loudspeaker may have different spatial emissions compared to the actual conditions of the hospital environment. However, this inherent bias of laboratory conditions was the same in all scenarios.

Some studies showed that the material has more impact on sound transmission than the geometric shape and design of the mask [31, 32]. It seems that in order to improve some characteristics affecting the protection efficiency and usability of face masks, new technologies should be used. It is proposed that nanofiber-based media have a high ratio of surface area to volume, low-pressure loss, high interconnectivity of voids, and controllable morphology which can be employed to achieve excellent filtering and possibly verbal communication and even personal thermal comfort in the process of producing newer face masks $[32,33]$.
Mheidly et al. [34] stated that during the current COVID19 pandemic, the design of effective strategies and increased skills in verbal communication with face masks is essential. It is proposed that hand gestures or body movements be employed by nurses wearing face masks to increase speech comprehensibility during interpersonal communication. The findings verified that background noise levels have a considerable influence on the ability to understand the speech of Persian words. Based on the observed critical role of background noise on speech intelligibility, some technical intervention or acoustic treatment must be applied in the noisy hospital environments for reducing reflecting noise from surfaces and noise sources. Finally, it is recommended that the face masks with the minimum required protection criteria while maintaining acceptable speech intelligibility should be worn by nurses employed in noisy healthcare settings.

\section{Conclusion}

Speech communications in healthcare settings are always challenging while wearing face masks. The current findings verified that the combination of face masks and the presence of background noise negatively impacts verbal communication in Persian. The high background noise in the healthcare settings can aggravate the effect sizes of the face masks on speech comprehensibility. Moreover, the vocal efforts of nurses when wearing N95 and N99 respirators were not acceptable even for a short conversation. Newer technology in this field as nanofiber-based media can be employed to facilitate excellent filtering, breathability, and consequently possible good verbal communication. The current experimental evidence can help professionals design practical guidelines to preserve nurses' verbal communication when using commercially available face masks during the COVID-19 pandemic.

Acknowledgements This study was supported by the Vice-Chancellor of Research and Technology of Hamadan University of Medical Sciences (Grant Number: 9905143043). We would like to thank the nurses of the healthcare settings for their active cooperation and participation in this research.

\section{References}

1. Magee, M., Lewis, C., Noffs, G., Reece, H., Chan, J., Zaga, C.J., et al.: Effects of face masks on acoustic analysis and speech perception: implications for peri-pandemic protocols. J. Acoust. Soc. Am. 148, 3562 (2020). https://doi.org/10.1121/10.0002873

2. Goldin, A., Weinstein, B.E., Shiman, N.: How do medical masks degrade speech perception? Hear. Rev. 27(5), 8-9 (2020)

3. Chughtai, A.A., Stelzer-Braid, S., Rawlinson, W., Pontivivo, G., Wang, Q., Pan, Y., et al.: Contamination by respiratory viruses on 
outer surface of medical masks used by hospital healthcare workers. BMC Infect. Dis. 19, 491 (2019)

4. Nickell, L.A., Crighton, E.J., Tracy, C.S., Al-Enazy, H., Bolaji, Y., Hanjrah, S., et al.: Psychosocial effects of SARS on hospital staff: survey of a large tertiary care institution. Can. Med. Assoc. J. 170, 793-798 (2004)

5. Andrade, E.L., Silva, D.C.C., Lima, E.A., Oliveira, R.A., Zannin, P.H.T., Martins, A.C.G.: Environmental noise in hospitals: a systematic review. Environ. Sci. Pollut. Res. 28, 19629-19642 (2021)

6. Darbyshire, J.L., Young, J.D.: An investigation of sound levels on intensive care units with reference to the WHO guidelines. Crit. Care 17(5), R187 (2013). https://doi.org/10.1186/cc12870

7. Pai, J.Y.: A study in hospital noise-a case from Taiwan. Int. J. Occup. Saf. Ergon. 13, 83-90 (2007)

8. Corey, R.M., Jones, U., Singer, A.C.: Acoustic effects of medical, cloth, and transparent face masks on speech signals. J. Acoust. Soc. Am. 148(4), 2371 (2020). https://doi.org/10.1121/10.0002279

9. Corey, R.M., Jones, U., Singer, A.C.: Comparison of the acoustic effects of face masks on speech. Hear. J. 5, 36-39 (2021)

10. Chmelík, V., Urbán, D., Zelem, L., Rychtáriková, M.: Effect of mouth mask and face shield on speech spectrum in Slovak Language. Appl. Sci. 11, 4829 (2021). https://doi.org/10.3390/ app11114829

11. Yi, H., Pingsterhaus, A., Song, W.: Effects of wearing face masks while using different speaking styles in noise on speech intelligibility during the COVID-19 Pandemic. Front. Psychol. 12, 682677 (2021). https://doi.org/10.3389/fpsyg.2021.682677

12. Wittum, K.J.: The effects of surgical masks on speech perception in noise. The Ohio State University. Research Thesis (2013)

13. Randazzo, M., Koenig, L.L., Priefer, R.: The effect of face masks on the intelligibility of unpredictable sentences. 179th Meeting of the Acoustical Society of America (2020)

14. Baldner, E.F., Doll, E., Mersbergen, M.R.: A review of measures of vocal effort with a preliminary study on the establishment of a vocal effort measure. J. Voice 29(5), 530-541 (2015)

15. Ribeiro, V.V., Dassie-Leite, A.P., Pereira, E.C., Santos, A.D.N., Martins, P., Irineu, R.A.: Effect of wearing a face mask on vocal self-perception during a pandemic. J. Voice (2020). https://doi.org/ 10.1016/j.jvoice.2020.09.006

16. Leer, V., Mersbergen, V.: Using the BORG CR10 physical exertion scale to measure patient perceived vocal effort pre and post treatment. J. Voice 31(3), 19-25 (2017). https://doi.org/10.1016/j. jvoice.2016.09.023

17. ISO 8253-1: Acoustics: Audiometric Test methods. Basic Pure Tone Air and Bone Conduction Threshold Audiometry. International Organization for Standardization, Geneva (2010)

18. ISO 8253-3: Acoustics: Audiometric Test Methods Part 3: Speech Audiometry. International Organization for Standardization, Geneva (2012).

19. IEC 60268-16: Standard. Sound System Equipment-Part 16: Objective Rating of Speech Intelligibility by Speech Transmission Index. International Electrotechnical Commission (2020)

20. Karami, M., Aliabadi, M., Golmohammadi, R., Hamidi, Nahrani, M.: The effect of hearing protection devices on speech intelligibility of Persian employees. BMC Res. Notes 13(529), 2-6 (2020)
21. Williams, N.: The Borg rating of perceived exertion (RPE) scale, questionnaire review. J. Occup. Med. 67, 404-405 (2017)

22. Fiorella, M.L., Cavallaro, G., Nicola, V.D., Quaranta, N.: Voice differences when wearing and not wearing a surgical mask. J. Voice (2021). https://doi.org/10.1016/j.jvoice.2021.01.026

23. Toscano, J.C., Toscano, C.M.: Effects of face masks on speech recognition in multi-talker babble noise. PLoS ONE 16(2), e0246842 (2021). https://doi.org/10.1371/journal.pone.0246842

24. Rahimi, Z., Shirali, G.A., Araban, M., Mohammadi, M.J., Cheraghian, B.: Mask use among pedestrians during the Covid-19 pandemic in Southwest Iran: an observational study on 10,440 people. BMC Public Health 21, 133 (2021). https://doi.org/10.1186/ s12889-020-10152-2

25. Round, M., Isherwood, P.: Speech intelligibility in respiratory protective equipment-implications for verbal communication in critical care. Trends Anaesth. Crit. Care 36, 23-29 (2021)

26. JiChoi, Y.: Acoustical measurements of masks and the effects on the speech intelligibility in university classrooms. Appl. Acoust. 180, 108145 (2021). https://doi.org/10.1016/j.apacoust.2021.108145

27. Atcherson, S.R., McDowell, B.R., Howard, M.P.: Acoustic effects of non-transparent and transparent face coverings. J. Acoust. Soc. Am. 149, 2249 (2021). https://doi.org/10.1121/10.0003962

28. Homans, N.C., Vroegop, J.L.: The impact of face masks on the communication of adults with hearing loss during COVID-19 in a clinical setting. Int. J. Audiol. (2021). https://doi.org/10.1080/ 14992027.2021.1952490

29. Smiljanic, R., Keerstock, S., Meemann, K., Ransom, S.M.: Face masks and speaking style affect audio-visual word recognition and memory of native and non-native speech. J. Acoust. Soc. Am. 149(6), 4013 (2021). https://doi.org/10.1121/10.0005191

30. Karagkouni, O.: The effects of the use of protective face mask on the voice and its relation to self-perceived voice changes. J. Voice (2021). https://doi.org/10.1016/j.jvoice.2021.04.014

31. Balamurali, B.T., Enyi, T., Clarke, C.J., Harn, S.Y., Chen, J.M.: Acoustic effect of face mask design and material choice. Acoust. Aust. (2021). https://doi.org/10.1007/s40857-021-00245-2

32. Essa, W.K., Yasin, S.A., Saeed, I.A., Ali, G.: Nanofiber based face masks and respirators as COVID-19 protection: a review. Membranes 11, 250 (2021). https://doi.org/10.3390/ membranes 11040250

33. Yang, A., Cai, L., Zhang, R., Wang, J., Hsu, P.C., Wang, H., et al.: Thermal management in nanofiber-based face mask. Nano Lett. 17, 35063510 (2017). https://doi.org/10.1021/Acs.Nanolett.7b00579

34. Mheidly, N., Fares, M.Y., Fares, Z.H.J.: Effect of face masks on interpersonal communication during the COVID-19 pandemic. Front. Public Health 8, 582191 (2020). https://doi.org/10.3389/ fpubh.2020.582191

Publisher's Note Springer Nature remains neutral with regard to jurisdictional claims in published maps and institutional affiliations. 